\title{
Des acteurs clés de la régulation de la sécrétion de GnRH : les cellules gliales et endothéliales de l'hypothalamus
}

\author{
par Jean-Claude Beauvillain \& Vincent Prévot \\ Unité Inserm 422, IMPRT 114, Service d'imagerie de l'Université de Lille 2, Place de Verdun, 59045 Lille cedex. \\ Adresser la correspondance à : Dr Jean-Claude Beauvillain, INSERM U 422, Place de Verdun, 59045 Lille cedex. \\ E-mail : jcb@lille.inserm.fr.
}

Reçu le 8 décembre 2003

\section{RÉSUMÉ}

De nombreux travaux publiés ces dix dernières années démontrent que la sécrétion de GnRH est susceptible d'être modulée par des facteurs provenant de cellules gliales mais aussi de l'endothélium des capillaires du plexus porte. Parmi les facteurs gliaux susceptibles d'intervenir, on peut distinguer le TGF $\beta$ qui peut agir directement sur les cellules à GnRH et les EGFs qui exercent leurs actions clairement stimulantes via la sécrétion de prostaglandine par les cellules astrocytaires voisines. Pour l'endothélium, il a été montré qu'il pouvait favoriser la libération de GnRH via la sécrétion de NO. En parallèle à ces actions, la glie et l'endothélium sont capables de moduler la sécrétion de GnRH en favorisant l'accès des terminaisons nerveuses à l'espace péricapillaire afin de faciliter le déversement de la neurohormone dans le sang porte. De fait, les terminaisons ne sont en situation de pouvoir déverser leur contenu que l'après midi du prooestrus, période où un pic de GnRH est nécessaire afin de provoquer le pic préovulatoire de $\mathrm{LH}$. Cette observation suggère qu'il existe une plasticité morphologique de l'éminence médiane mettant en cause des mouvements des tanycytes (épendymocytes de l'éminence médiane). Cette plasticité peut être induite par des facteurs de croissances gliaux (TGF $\beta$ et TGF $\alpha$ ) et par le NO d'origine endothéliale. Au site de libération de la GnRH, l'interaction neuro-glio-endothéliale apparaît donc un élément clé de la régulation de sécrétion de GnRH.

\section{SUMMARY Hypothalamic glial cells and endothelial cells as key regulators of GnRH secretion}

During the last decade, compelling evidence has been provided that, in addition of being regulated by transsynaptic inputs, GnRH neuroendocrine secretion is modulated by factors released both by glial cells and the endothelium of pituitary portal blood vessels. Glial cells exert their regulatory influence on GnRH release through the secretion of growth factors, such as TGF $\beta$ s and peptides member of the EGF family, that act either directly on GnRH neurons or require prostaglandin release from astrocytes, respectively. On the other hand vascular endothelial cells stimulate GnRH release via NO secretion. In addition, recent studies suggest that both glial cells and endothelial cells of the median eminence can modulate the direct access of GnRH neuroendocrine terminals to the vascular wall and thus control GnRH release efficiency. During the reproductive cycle, direct neurovascular contacts of GnRH nerve endings, that are engulfed in tanycytic endfeet, only occur at periods when massive GnRH release is required, i.e., at the onset of the preovulatory $\mathrm{GnRH} / \mathrm{LH}$ surge on the day of proestrus. Recent in vitro and in vivo data demonstrate that both glial (TGF $\alpha$ and TGF $\beta$ ) and endothelial (NO) factors can induce such morphological plasticity. Neuro-glio-endothelial interactions at the median eminence of the hypothalamus thus appear to be key regulatory mechanisms for GnRH neuroendocrine secretion.
La fonction de reproduction est contrôlée au niveau du système nerveux central par une neurohormone appelée la GnRH (gonadotropin releasing hormone) qui est synthétisée par un petit nombre de cellules spécialisées dispersées, chez les Rongeurs, dans l'aire préoptique de l'hypothalamus.
La sécrétion de GnRH dans le système porte hypophysaire est régulée par de nombreux signaux qui peuvent opérer soit au niveau du corps cellulaire ou au niveau des terminaisons nerveuses localisées dans l'éminence médiane. Pour beaucoup, ces signaux sont d'origine neuronale agissant par voie trans-synaptique ou par 
action de type paracrine (Smith \& Jennes, 2001; Ojeda \& Terasawa, 2002). Par ailleurs, il est évident que les hormones stéroïdiennes gonadiques ont une influence majeure sur l'activité des cellules à GnRH. Cette influence semble s'exercer majoritairement de façon indirecte via des récepteurs aux œstrogènes de type $\alpha$ (Herbison, 1998) mais elle pourrait aussi s'exercer de façon directe via des récepteurs de type $\beta$ (Herbison \& Pape, 2001). Cependant, depuis un peu plus de dix années, sont apparus de nombreux travaux démontrant que les cellules gliales avaient un rôle important dans le contrôle de la sécrétion de $\mathrm{GnRH}$ (revues dans Ojeda \& Terasawa, 2002; Dhanpapani et al., 2003). Effectivement, il semblait logique que ces cellules puissent assurer ce genre de fonction dans la mesure où elles expriment une variété de substances capables d'influencer l'activité des neurones (prostaglandines, glutamate, facteurs de croissances, AMP cyclique, etc.). Concernant la régulation de la sécrétion de $\mathrm{GnRH}$ par des facteurs gliaux, ce sont les effets des facteurs de croissance qui ont suscité le plus de travaux, actuellement les plus aboutis. Parmi eux on distingue le TGF $\beta 1$ agissant via des récepteurs sérine thréonine kinase et des membres de la famille des "epidermal growth factors" (EGF), agissant via des récepteurs de type tyrosine kinase.

En plus de cette régulation d'origine gliale, des travaux menés par Prévot et al. (1998, 1999b, 2000b) ont montré que l'endothélium des capillaires du système porte était capable de sécréter des substances susceptibles d'intervenir sur la libération de $\mathrm{GnRH}$. Plus précisément, du monoxyde d'azote d'origine endothéliale (NO) apparaît capable de stimuler la sécrétion de $\mathrm{GnRH}$ dans des délais rapides suggérant un rôle jusqu'alors insoupçonné de cet endothélium sur la modulation de la sécrétion de certaines neurohormones.

En dehors des rôles modulateurs de la glie et/ou de l'endothélium via la sécrétion de substances agissant par des actions juxtacrines, il est apparu, grâce à des études ultrastructurales très précises, que des phénomènes de plasticité morphologique impliquant des mouvements de la glie au niveau de l'éminence médiane pouvaient favoriser ou non l'accessibilité des terminaisons nerveuses à GnRH à l'espace péricapillaire (Prévot et al.,1999a). Ce type de plasticité apparaît par conséquent comme un autre mode de contrôle de la sécrétion de GnRH par les cellules gliales.

Dans cette revue, nous ferons donc le point successivement sur l'effet du TGF $\beta_{1}$, des EGFs, et du NO sur la libération de $\mathrm{GnRH}$, et nous aborderons enfin l'implication de la plasticité morphologique sur le fonctionnement du système à GnRH.

\section{TGF $\beta$ ET GnRH}

La notion d'une action stimulante directe du TGF $\beta$ sur la libération de $\mathrm{GnRH}$ est née à partir d'observations de co-cultures de cellules $\mathrm{GT}_{1}$ (cellules à $\mathrm{GnRH}$ immortalisées) avec des astrocytes (Melcangi et al., 1995;
Buchanan et al., 2000). Faisant suite à ces observations il est apparu que le milieu de culture des astrocytes était capable aussi de stimuler la sécrétion de GnRH à condition de le chauffer préalablement suggérant que le facteur susceptible d'agir était synthétisé sous une forme inactive pouvant être activée par des traitements exogènes. Parmi les facteurs envisagés, le TGF $\beta$ était un bon candidat dans la mesure où cette molécule était effectivement exprimée par des astrocytes (Constam et al., 1992) et qu'elle pouvait être libérée, associée avec une protéine porteuse la rendant biologiquement inactive (Lawrence et al., 1985). L'utilisation d'anticorps dirigés contre le TGF $\beta$ dans le milieu de culture a permis de confirmer cette hypothèse dans la mesure où cette immunoneutralisation abolit l'effet stimulant des astrocytes sur la sécrétion de GnRH (Melcangi et al., 1995). Il est apparu ensuite que le TGF $\beta$ avait également un effet sur l'expression des ARNm codant pour la GnRH dans les cellules GT, surtout pendant les six premières heures après traitement (Galbiati et al., 1996). Cependant cet effet sur cellules GT1 n'indiquait pas obligatoirement que le TGF $\beta$ agissait in vivo sur les cellules à $\mathrm{GnRH}$. Un pas supplémentaire a été franchi grâce aux études faites sur des coupes d'aires préoptiques de rat montrant que les cellules à GnRH contiennent le récepteur au TGF $\beta$ de type 1 (Prévot et al., 2000a) suggérant que les cellules à GnRH peuvent effectivement répondre à la stimulation de l'activine et problablement au TGF $\beta$. Reste qu'actuellement, in vivo, l'effet du TGF $\beta$ sur la libération de GnRH n'est pas très claire. Ce qui a été rapporté jusqu'à maintenant c'est que le TGF $\beta$ ne modifiait pas la libération de GnRH lorsqu'il était ajouté à des explants d'éminences médianes déconnectées de l'aire préoptique (Ojeda et al., 1990).

\section{EGF ET GnRH}

Les travaux sur les EGF ont été entrepris pour la grande majorité dans le laboratoire de Sergio Ojeda (Revue dans Ojeda et Terasawa, 2002). Le TGF $\alpha$ et les neurégulines, deux membres de cette famille, apparaissent impliqués dans la régulation de la sécrétion de $\mathrm{GnRH}$. Ce sont essentiellement des travaux effectués sur l'initiation de la puberté chez les femelles qui ont permis de disséquer les modes d'action de ces substances. Rappelons que le TGF $\alpha$ agit via les récepteurs $\operatorname{erbB}_{1}$ alors que les neurégulines agissent via les récepteurs erbB $\mathrm{B}_{4}$. En fait il a été récemment démontré (revue dans Yarden \& Sliwowski, 2001) que le récepteur erbB $\mathrm{B}_{2}$ était impliqué aussi dans les actions de ces EGFs en tant que co-récepteur et que l'hétérodimérisation amplifiait la stimulation.

Une première expérience majeure concernant l'effet du TGFo., menée par Ojeda et al. (1990), a consisté à montrer que cette molécule était capable de stimuler la sécrétion de $\mathrm{GnRH}$ à partir de fragments d'éminences médianes. Cette action passe par la sécrétion de prostaglandines puisque l'inhibition de sa synthèse abolit 
l'effet du TGF $\alpha$. Des travaux effectués par la suite sur des cellules GT1 montrent que le TGF $\alpha$ n'a aucune action sur la libération de GnRH sur cette lignée, ce qui s'explique d'ailleurs par le fait que ces cellules ne possèdent pas de récepteurs erbBl (Ma et al., 1994; Voigt et al., 1996). Par contre, lorsque l'on traite des cellules astrocytaires avec du TGF $\alpha$, on provoque une libération très importante de prostaglandine. En résumé, le TGF $\alpha$ synthétisé par les cellules astrocytaires (Junier et al., 1991) agit sur les récepteurs erbB, présents dans les cellules gliales (Ma et al., 1994) via une action paracrinejuxtacrine et provoque une libération de prostaglandine qui va alors stimuler la sécrétion de $\mathrm{GnRH}$ par les terminaisons nerveuses qui possèdent les récepteurs aux prostaglandines. Par conséquent, et contrairement à ce qui est observé pour le TGF $\beta$, le TGF $\alpha$ n'agit pas directement sur les cellules à GnRH.

Les différents travaux menés dans l'équipe d'Ojeda ont très bien démontré l'implication du TGF $\alpha$ et des récepteurs erbB $B_{1}$ dans l'apparition de la puberté et la quantification de ces deux substances montre des variations d'expression très significatives aux périodes critiques de la maturation sexuelle. Cette implication a été récemment confirmée par le fait qu'une mutation du

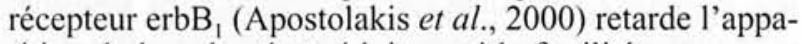
rition de la puberté et réduit aussi la fertilité.

En dehors du TGF $\alpha$ les neurégulines 1 et 3 ainsi que deux de leurs récepteurs, erbB $\mathrm{B}_{2}$ et $\operatorname{erbB}_{4}$, semblent être impliqués dans la régulation de la $\mathrm{GnRH}$ dans la mesure où ces substances montrent aussi des taux d'expression très différents dans l'hypothalamus au cours des différentes phases de la maturation sexuelle (Ma et al., 1999).

Les neurégulines 1 et 3 ainsi que les récepteurs erbB ${ }_{4}$ et erbB $\mathrm{B}_{2}$ sont exprimés dans les astrocytes hypothalamiques de différentes régions notamment celles impliquées dans la régulation de la GnRH. Le mode d'action des neurégulines sur la sécrétion de GnRH est semblable à celui du TGF $\alpha$ et passe via la sécrétion de prostaglandine après une activation des récepteurs erbB $B_{4}$. L'importance du récepteur erbB $\mathrm{B}_{4}$ a été très récemment démontrée chez un modèle de souris transgéniques chez lequel le gène de ce récepteur a été invalidé spécifiquement dans les astrocytes (Prévot et al., 2003a). Les souris porteuses de ce transgène ont un retard de puberté mais aussi une capacité de reproduction significativement diminuée chez les femelles primipares. Ce retard est à mettre en relation avec une diminution de la sécrétion de GnRH (Prévot et al., 2003a). L'ensemble de ces travaux sur les EGFs démontre donc de façon évidente que les astrocytes sont des cellules fortement impliquées dans la régulation de la sécrétion de $\mathrm{GnRH}$ au niveau de l'éminence médiane.

\section{MONOXYDE D'AZOTE (NO) D'ORIGINE ENDOTHÉLIALE ET GnRH}

L'idée que du NO d'origine endothéliale puisse intervenir sur la libération de GnRH est issue d'observations faites sur des éminences médianes maintenues en survie
(Prévot et al., 1998). Dans ce modèle expérimental, il a pu être montré, grâce à l'utilisation d'une méthode ampérométrique permettant de mesurer en temps réel la libération de NO, qu'une induction de sécrétion de ce gaz par différentes substances était capable de stimuler très rapidement la sécrétion de GnRH. Il a été constaté que cette induction était inhibée par du L-NIO, un inhibiteur de la NOS (nitric oxide synthase), enzyme nécessaire à la fabrication du NO, à des concentrations faibles, suggérant que cette NOS était de type endothélial (Prévot et al., 1999b). Cette hypothèse était compatible avec le fait qu'une grande quantité de NOS est contenue dans l'endothélium des capillaires du plexus porte, à proximité immédiate des terminaisons nerveuses à GnRH (Prévot et al., 2000b). Parmi les substances capables d'intervenir sur la sécrétion du NO, la plus intéressante par rapport à la sécrétion de GnRH semble être l'œstradiol qui induit la fabrication de ce neurotransmetteur gazeux par l'éminence médiane dans les dix minutes suivant son addition dans le milieu de survie (Prevot et al., 1999b). Ces résultats suggèrent que cette action pourrait être membranaire. Cette hypothèse est par ailleurs étayée par le fait qu'une action comparable est obtenue en utilisant de l'œstradiol conjugué à la BSA qui est incapable de passer la membrane cytoplasmique. Ces résultats nous laissent donc penser qu'il peut exister une action rapide, non génomique, de l'œstradiol qui pourrait intervenir dans la sécrétion de GnRH. Cependant, à côté de ces observations, d'autres travaux montrent qu'il existe aussi une action génomique de l'œstradiol sur la formation du $\mathrm{NO}$, avec des arguments encore plus précis quant à l'origine endothéliale. Effectivement, l'analyse par Western blot des concentrations de NOS endothéliale dans l'éminence médiane au cours du cycle oestral révèle que celles ci varient selon les stades du cycle avec une valeur maximale le jour du proøstrus (Knauf et al., 2001a). Cette augmentation est d'ailleurs liée à la présence d'œstradiol, comme des travaux complémentaires ont pu le montrer (Knauf et al., 2001a)). Ces expériences suggèrent donc qu'une sécrétion de NO par l'endothélium peut varier au cours du cycle œstral et ceci est effectivement le cas puisque la mesure en temps réel du NO montre que celui ci est libéré de façon pulsatile par l'éminence médiane avec une fréquence proche de la pulsatilité de libération de $\mathrm{GnRH}$ mais surtout que l'amplitude des pulses de $\mathrm{NO}$ est très significativement augmentée le jour du proœstrus (Knauf et al., 200lb). Ces travaux démontrent donc l'implication du NO d'origine endothéliale sur la sécrétion pulsatile et cyclique de $\mathrm{GnRH}$. Ces résultats sont complètement en accord avec ceux de Aguan et al. (1996) démontrant que l'injection intracérébroventriculaire d'un oligonucléotide anti-sens de la NOS endothéliale prévient l'obtention du pic de $\mathrm{GnRH} / \mathrm{LH}$ induit par l'œstradiol.

En conclusion, ces travaux effectués sur le NO fournissent donc une somme d'arguments en faveur d'un rôle de l'endothélium du système porte dans la modulation d'un type de sécrétion neurohormonale, la GnRH. Il est très probable que d'autres substances sécrétées par l'endothélium interviennent aussi sur cette modulation, 
de même qu'il est très vraisemblable que d'autres systèmes neurohormonaux puissent aussi être concernés par ce type de régulation.

\section{PLASTICITÉ MORPHOLOGIOUE DE L'ÉMINENCE MÉDIANE ET GnRH}

L'observation des terminaisons nerveuses à $\mathrm{GnRH}$ de l'éminence médiane, notamment à l'échelle ultrastructurale, a très vite permis de constater que celles ci étaient localisées relativement loin de l'espace péricapillaire, quel que soit le sexe (Koslowski \& Coates, 1985). En fait, ces terminaisons sont entourées par des prolongements tanycytaires formant une barrière entre elles et l'espace péricapillaire. Cette situation posait par conséquent le problème du passage de la GnRH dans les capillaires du plexus porte. Cette constatation semblait paradoxale dans la mesure où les terminaisons nerveuses contenant d'autres neurohormones comme la somatostatine, le CRF, la GHRH, étaient très couramment observées au contact de la membrane basale parenchymenteuse bordant l'espace péricapillaire. Il a été alors imaginé que, pour les terminaisons à $\mathrm{GnRH}$, il pouvait exister, au moins chez la femelle, une plasticité morphologique de la zone externe de l'éminence médiane permettant l'accès des terminaisons nerveuses aux capillaires au moment où une sécrétion massive de GnRH était nécessaire. Ce sont les travaux de Prévot et al. (1999a), effectués à l'échelle ultrastructurale qui ont définitivement permis de répondre à cette question. Il a été effectivement constaté, chez la ratte femelle, après analyse des éminences médianes à différentes heures de chaque stade du cycle œstral, que certaines terminaisons nerveuses pouvaient être vues au contact des capillaires mais exclusivement l'après midi du proœstrus. Une étude quantitative après observation de très nombreuses coupes ultrafines sériées a permis de constater que $12 \%$ des terminaisons nerveuses étaient situées au contact des capillaires l'après midi du proœstrus alors qu'aucun contact n'avait pu être observé aux autres stades du cycle œstral. Par ailleurs, des images suggérant des mouvements de retrait des tanycytes pouvant parfois entraîner des évaginations de l'espace péricapillaire dans le parenchyme nerveux étaient fréquentes également l'après midi du proœstrus. L'ensemble de ces travaux suggère que les tanycytes pourraient subir l'influence de divers facteurs capables de modifier leurs formes afin de permettre l'accès des terminaisons nerveuses à l'espace péricapillaire à des moments bien précis du cycle œstral. Cette hypothèse est d'autant plus probable qu'il a pu être démontré récemment que les tanycytes en culture étaient susceptibles de s'étendre sous l'influence du TGF $\alpha$ ou de se rétracter sous l'influence du TGFß (Prévot et al., 2003b). Il paraît donc possible que ces deux facteurs puissent intervenir in vivo pour favoriser l'accessibilité des terminaisons nerveuses à l'espace péricapillaire. De plus des travaux du laboratoire (De Seranno et al., 2002) indiquent que le NO d'origine endothéliale, évoqué précédemment, pourrait aussi moduler la forme des tanycytes. Il parait donc clair que les tanycytes sont des acteurs majeurs du remaniement morphologique de la zone externe de l'éminence médiane au cours du cycle estral et que ce phénomène est vraisemblablement très important dans la régulation de la libération de $\mathrm{GnRH}$ dans le sang porte.

En conclusion générale, il est maintenant évident que les cellules gliales et les cellules endothéliales de l'éminence médiane ont un rôle important dans le fonctionnement du système à GnRH. Les interactions neurogliales peuvent s'exercer au niveau des corps cellulaires et de l'éminence médiane alors que le rôle des cellules endothéliales ne semble s'exercer qu'au niveau de l'éminence médiane. Actuellement certains facteurs comme le TGF $\alpha$, le TGF $\beta$ et le NO font l'objet de nombreuses investigations et leurs modes d'action apparaissent finalement plus complexes que l'on pouvait l'imaginer dans la mesure où des mêmes molécules peuvent avoir une action sur les terminaisons nerveuses (directe ou indirecte) mais aussi sur les tanycytes afin d'en modifier leurs formes. Il est très probable que, dans l'avenir, d'autres molécules provenant notamment des cellules endothéliales de l'éminence médiane seront incriminées dans la modulation de la sécrétion de GnRH.

\section{BIBLIOGRAPHIE}

Aguan K., Mahesh V. B., Ping L., Bhat G. \& Brann D. W., Evidence for a physiological role for nitric oxide in the regulation of LH surge: effect of central administration of antisense oligonucleotides to nitric oxide synthase. Neuroendocrinology, 1996, 64, 449-455.

Apostolakis E. M., Garai J., Lohmann J. E., Clark J. H. \& O’Malley B. W., Epidermal growth factor activates reproductive behavior independent of ovarian steroids in female rodents. Mol. Endocrinol., 2000, 14, 1086-1098.

Buchanan C. D., Mahesh V. B. \& Brann D. W.., Estrogen-astrocyte-luteinizing hormone-releasing hormone signalling: a role for transforming growth factor- $\beta$. Biol. Reprod., 2000. $62,1710-1721$.

Constam D. B., Philipp J., Malipiero U. V., Ten D. P, Shachner M. \& Fontana A., Differential expression of transforming growth factor- $\beta 1, \beta 2$, and $\beta 3$ by glioblastoma cells, astrocytes, and microglia. J. Immunol., 1992, 148, 1404-1410.

De Seranno S., Buée L., Beauvillain J. C., Prévot V., Glial-endothelial interactions may cause structural changes in the morphology of the tanycytes of the median eminence by inducing the remodelling of their cytosqueleton. $3^{\text {nd }}$ Forum of European Neuroscience Paris, 2002, 089.6.

Dhandapani K. M., Virendra B. M. \& Brann D. W., Astrocytes and brain function : implications for reproduction. Exp. Biol. Med., 2003, 228, 253-260.

Galbiati M., Zanisi M., Messi E., Cavaretta I., Martini L. \& Melcangi R. C., Transforming growth factor $\beta$ and astrocytic conditionned medium influence luteinizing hormone-releasing hormone gene expression in the hypothalamus cell line GT1. Endocrinology, 1996, 137, 5605-5609.

Herbison A. E., Multimodal influence of estrogen upon gonadotropin-releasing hormone neurons. Endocr. Rev., 1998, 19. 302-330

Herbison A. E. \& Pape J. R., New evidence for oestrogen receptors in gonadotropin-releasing hormone neurons. Frontiers In Neuroendocrinology, 2001, 22, 292-308. 
Junier M. P., Ma Y. J., Costa M. E., Hoffman G., Hill D. F. \& Ojeda S. R., Transforming growth factor $\alpha$ contributes to the mechanism by which hypothalamic injury induces precocious puberty. Proc. Natl. Acad. Sci. USA., 1991, 88, 9743-9747.

Knauf C., Ferreira S., Hamdane M., Maillot C., Prévot V., Beauvillain J. C. \& Croix D., Variation of endothelial nitric oxide synthase synthesis in the median eminence during the rat estrous cycle: an additional argument for the implication of vascular blood vessel in the control of GnRH release. Endocrinology, 2001a, 142, 4288-4294.

Knauf C., Prévot V., Stefano G. B., Mortreux G., Beauvillain J. C. \& Croix D., Evidence for a spontaneous nitric oxide release from the rat median eminence: influence on gonadotropin releasing hormone release. Endocrinology, 2001b, 142 , 2343-2350.

Koslowski G. P. \& Coates P. M., Ependymoneuronal specializations between LHRH fibers and cells of the cerebroventricular system. Cell Tissue Res., 1985, 242, 301-311.

Lauwrence D. A., Pircher R. \& Jullien P., Conversion of a high molecular weight latent beta-TGF from chicken embryo fibroblasts into a low molecular weight active beta TGF under acidic conditions. Biochem. Biophys. Res. Commun., $1985,198,327-336$.

Ma Y. J., Hill D. F., Creswick K. E., Costa M. E. \& Ojeda S. R., Neuregulins signaling via a glial erbB $\mathrm{B}_{2} / \mathrm{erbB}_{4}$ receptor complex contribute to the neuroendocrine control of mammalian sexual development. J. Neurosci., 1999, 19, 9913-9927.

Ma Y. J., Hill D. F., Junier M. P., Costa M. E., Felder S. E. \& Ojeda S. R., Expression of epidermal growth factor receptor changes in the hypothalamus during the onset of female puberty. Mol. Cell. Neurosci., 1994, 5, 246-262.

Melcangi R. C., Galbiati M., Messi E., Piva F., Martini L. \& Motta M., Type l astrocytes influence luteinizing hormonereleasing hormone release from the hypothalamic cell line GT1-1: is transforming growth factor- $\beta 1$ the principle involved? Endocrinology, 1995, 136, 679-686.

Ojeda S. R. \& Terasawa E. I., Neuroendocrine regulation of puberty. Hormones, Brain and Behavior, edited by D. Pfaff, A. Arnold, A. Etgen, S. Fahrbach \& R. Rubin, volume 4, 2002, 589-659.

Ojeda S. R., Urbanski H. F., Costa M. E., Hill D. F. \& Moholt-Siebert M., Involvement of transforming growth factor $\alpha$ in the release of luteinizing hormone-releasing hormone from the developing female hypothalamus. Proc. Natl. Acad. Sci. USA., 1990, 87, 9698-9702.

Prévot V., Bouret S., Croix D., Takumi T., Jennes L., Mitchell V.
\& Beauvillain J. C., Evidence that members of the TGF $\beta$ superfamily play a role in regulation of the $\mathrm{GnRH}$ neuroendocrine axis: expression of a type 1 threonine kinase receptor for TGF $\beta$ and activin in GnRH neurones and hypothalamic areas of the female rat. J. Neuroendocrinol., 2000a, $12,665-670$.

Prévot V., Bouret S., Stefano G. B. \& Beauvillain J. C., Median eminence nitric oxide signaling. Brain. Res. Rev. 2000b, 34. 27-41.

Prévot V., Cornea A., Mungenast A., Smiley G. \& Ojeda S. R., Activation of erbB-, signaling in tanycytes of the median eminence stimulates Transforming Growth Factor $\beta 1$ release via prostaglandin E2 production and induces cell plasticity. J. Neurosci., 2003b, 23, 10622-10632.

Prévot V., Croix D., Bouret S., Dutoit S., Tramu G., Stefano S. \& Beauvillain J. C., Definitive evidence for the existence of morphological plasticity in the external zone of the median eminence during the rat estrous cycle: implication of neuroglio- endothelial interactions in the gonadotropin-releasing hormone release. Neuroscience, 1999a, 94, 809-819.

Prévot V., Croix D., Rialas C. M., Poulain P., Fricchione G. L., Stefano G. B. \& Beauvillain J. C., Estradiol coupling to endothelial nitric oxide stimulates gonadotropin-releasing hormone release from rat median eminence via a membrane receptor. Endocrinology, 1999b, 140, 652-659.

Prévot V., Rialas C. M., Croix D., Salzet M., Dupouy J. P., Poulain P., Beauvillain J. C. \& Stefano G. B., Morphin and anandamide coupling to nitric oxide stimulates $\mathrm{GnRH}$ and CRF release from rat median eminence: neurovascular regulation. Brain Res., 1998, 790, 236-244.

Prévot V., Rio C., Gyeong J. C., Lomniczi A., Heger S., Neville C. M, Rosenthal N. A, Ojeda S. R. \& Corfas G., Normal female sexual development requires neuregulin-erbB receptor signaling in hypothalamic astrocytes. J. Neurosci., 2003a, 23, 230-239.

Smith M. J. \& Jennes L., Neural signals that regulate GnRH neurones directly during the oestrous cycle. Reproduction, 2001, $122,1-10$.

Voigt P., Ma Y .J., Gonzalez D., Fahrenbach W. H., Wetsel W. C., Berg von Der Emden K., Hill D. F., Taylor K. G., Costa M. E., Seidah N. G. \& Ojeda S. R., Neural- and glial-mediated effects of growth factors acting via tyrosine kinase receptors on luteinizing hormone-releasing hormone neurons. Endocrinology, 1996, 137, 2593-2605.

Yarden Y. \& Sliwkowski M. X., Untangling the ErbB signalling network. Nat. Rev. Mol. Cell. Biol., 2001, 2, 127-137. 